\title{
MEASUREMENT AND CORRECTION OF LINEAR OPTICS AND COUPLING AT TEVATRON COMPLEX
}

Valeri Lebedev*, Vladimir Nagaslaev and Alexander Valishev

*Fermilab, MS 340, PO Box 500, Batavia, IL 60510

Vadim Sajaev

ANL, Argonne, IL 60439, USA

Abstract

The optics measurements have played important role in improving the performance of Tevatron collider. Until recently, most of them were based on the differential orbit measurements with data analysis, which neglects measurement inaccuracies such as differences in differential responses of beam position monitors, their rolls, etc. To address these complications we have used a method based on the analysis of many differential orbits. That creates the redundancy in the data allowing to get more detailed understanding of the machine. In this article we discuss the progress with Tevatron optics correction, its present status and future improvements.

\section{Introduction}

The commissioning of Tevatron Run II began in the spring of 2001 with the first luminosity seen in June. By the year's end the luminosity was in the range of (5-10) $10^{30} \mathrm{~cm}^{-2} \mathrm{~s}^{-1}$. Although the luminosity growth was significantly slower than expected, steady growth of luminosity has been demonstrated during last three years with the peak luminosity of $1.02 \cdot 10^{32} \mathrm{~cm}^{-2} \mathrm{~s}^{-1}$ achieved in July 2004. This luminosity growth would not be possible without thorough understanding of the accelerator physics problems, which have restricted the machine operation. Understanding and tuning the Tevatron optics was one of the main contributors to the success. Two problems have been encountered: the emittance growth due to optics mismatch at injection, and optics mismatch for the collision optics with subsequent increase of beta-functions in the interaction points (IP).

The collider is filled from Main Injector (MI) by protons and antiprotons through two different transfer lines. To maximize the luminosity the optics of each line has to be matched to both rings. Although, in principle, emittance growth related to each transfer could be measured using the emittance monitors of each ring, in reality, this method does not work because of uncertainty in calibration of emittance monitors. To exclude this uncertainty the round trip emittance measurement has been used. In this case the proton beam is sent from MI to Tevatron through the proton transfer line and then sent back to MI through the antiproton line. The MI emittance monitor is used to measure the total emittance growth for both transfers. The measurements performed in 2002 exhibited the round trip emittance growth of about 50\%. After linear optics correction in both transfer lines this value was only slightly improved and still stayed well above the emittance growth related to the betatron oscillations due to injection errors. Initial estimates of possible coupling effects yielded that small coupling cannot be a reason of such emittance growth. Soon we learned that the coupling in Tevatron is not small and leads to the significant emittance growth. The source of the coupling has been the regular skew-

\footnotetext{
* Work supported by the Universities Research Assos., Inc., under contract DE-AC02-76CH03000 with the U.S. Dept. of Energy.

- Work supported by U. S. Department of Energy, Office of Basic Energy Sciences under Contract No. W-31-109ENG-38.
} 
quadrupole component in all Tevatron dipoles. It originated from settling down the superconducting coil relative to the iron core due to compression of thermo-insulating coil support by $\sim 150 \mu \mathrm{m}$. Although the value of skew quadrupole gradient, $G_{s}$, for a single dipole does not look large $\left(G_{s} A / B_{0} \sim(1.5-2) \cdot 10^{-4}\right.$, where $A=2.54 \mathrm{~cm}$ and $B_{0}$ is the dipole field) summing the contributions of all 772 dipoles yields the uncompensated tune split $\sim 0.3$. This value is usually compensated to better than $5 \cdot 10^{-3}$ by machine skew quadrupoles but it still leaves large local coupling through the entire machine. 112 dipoles, which did not have nearby machine skew quadrupoles, were main source of this local coupling. During the 2003 shutdown their skew quadrupole fields were corrected by restoring thickness of the thermo-insulating supports (shimming of the dipoles). That resulted in a significant reduction of $x-y$ coupling on the emittance growth.

Optics measurements performed in 2003 showed that the Tevatron low-beta optics has been strongly mismatched. That resulted in a beta-wave through the entire machine and increased beta-functions in IPs with the corresponding luminosity loss of $\sim 15-20 \%$. Optics correction in IPs was performed in the spring of 2004. It also yielded some reduction of optics mismatch in arcs but further improvements are still required.

\section{Emittance growth due to $X-Y$ coupling}

To find the emittance growth due to $\mathrm{X}-\mathrm{Y}$ coupling at injection we will parameterize the eigen-vectors of coupled betatron motion in the following form:

$$
\mathbf{v}_{1}=\left[\begin{array}{c}
\sqrt{\beta_{1 x}} \\
-\frac{i(1-u)+\alpha_{1 x}}{\sqrt{\beta_{1 x}}} \\
\sqrt{\beta_{1 y}} e^{i v_{1}} \\
i u+\alpha_{1 y} \\
-\frac{{ }^{\beta_{1 y}}}{} e^{i v_{1}}
\end{array}\right], \quad \mathbf{v}_{2}=\left[\begin{array}{c}
\sqrt{\beta_{2 x}} e^{i v_{2}} \\
-\frac{i u+\alpha_{2 x}}{\sqrt{\beta_{2 x}}} e^{i v_{2}} \\
\sqrt{\beta_{2 y}} \\
-\frac{i(1-u)+\alpha_{2 y}}{\sqrt{\beta_{2 y}}}
\end{array}\right] .
$$

where $\beta_{n x, n y}$ and $\alpha_{n x, n y}$ are the generalized beta- and alpha-functions, and parameters $u, v_{1}$ and $v_{2}$ are determined by the symplecticity conditions. Then, the particle motion can be written as

$$
\mathbf{x}=\frac{1}{2}\left(\sqrt{2 \varepsilon_{1}}\left(\mathbf{v}_{1} e^{i \mu_{1}}+\mathbf{v}_{1}{ }^{*} e^{-i \mu_{1}}\right)+\sqrt{2 \varepsilon_{2}}\left(\mathbf{v}_{2} e^{i \mu_{2}}+\mathbf{v}_{2}{ }^{*} e^{-i \mu_{2}}\right)\right)
$$

where $\varepsilon_{1}$ and $\varepsilon_{2}$ are the rms single particle emittances, and $\mu_{1}$ and $\mu_{2}$ are the betatron phase advances. Multiplying each side of Eq. (2) by $\mathbf{v}_{1}^{+} \mathbf{U}$ or $\mathbf{v}_{2}{ }^{+} \mathbf{U}$ and using orthogonality conditions for eigen-vectors we obtain

$$
\varepsilon_{1}=\frac{1}{2}\left(\mathbf{v}_{1}{ }^{+} \mathbf{U} \mathbf{x}\right)^{2}, \quad \varepsilon_{2}=\frac{1}{2}\left(\mathbf{v}_{2}{ }^{+} \mathbf{U} \mathbf{x}\right)^{2} .
$$

Here “+” denotes the Hermite conjugate vector, and $\mathbf{U}$ is the symplectic unit matrix,

$$
\mathbf{U}=\left[\begin{array}{ll}
0 & \mathbf{u} \\
\mathbf{u} & 0
\end{array}\right] \quad, \quad \mathbf{u}=\left[\begin{array}{cc}
0 & 1 \\
-1 & 0
\end{array}\right]
$$

Table 1. Twiss parameters at proton and antiproton injection points before shimming of dipoles

\begin{tabular}{|l|c|c|c|c|}
\hline & \multicolumn{2}{|c|}{ Proton injection } & \multicolumn{2}{c|}{ Antiproton injection } \\
\hline & Mode 1 & Mode 2 & Mode 1 & Mode 2 \\
\hline$\beta_{x}$ & $102.0 \mathrm{~m}$ & $4.71 \mathrm{~m}$ & $84.34 \mathrm{~m}$ & 4.04 \\
\hline$\alpha_{x}$ & -0.8348 & -0.0321 & -0.6825 & -0.03233 \\
\hline$\beta_{y}$ & $2.96 \mathrm{~m}$ & $65.42 \mathrm{~m}$ & $3.77 \mathrm{~m}$ & $75.64 \mathrm{~m}$ \\
\hline$\alpha_{y}$ & 0.0126 & 0.2019 & 0.0294 & 0.5227 \\
\hline$v$ & $-110.9^{0}$ & $-112.47^{0}$ & $-128.5^{0}$ & $-126.9^{0}$ \\
\hline$u$ & \multicolumn{2}{|c|}{-0.0609} & \multicolumn{2}{|c|}{.0539} \\
\hline
\end{tabular}


For Gaussian distribution of the injected beam its distribution function at the injection point can be written in the following form

$$
f(\mathbf{x})=\frac{1}{4 \pi^{2} \varepsilon_{1} \varepsilon_{2}} \exp \left(-\frac{1}{2} \mathbf{x}^{T} \boldsymbol{\Xi} \mathbf{x}\right),
$$

where matrix $\boldsymbol{\Xi}$ is determined by the eigen-vectors, $\mathbf{v}_{t 1}$ and $\mathbf{v}_{t 2}$, of the incoming beam

$$
\begin{aligned}
& \boldsymbol{\Xi}=\mathbf{U} \mathbf{V}_{t} \boldsymbol{\Xi}^{\prime} \mathbf{V}_{t}^{T} \mathbf{U}, \quad \boldsymbol{\Xi}^{\prime}=\left[\begin{array}{cc}
\varepsilon_{1}^{-1} \mathbf{I} & 0 \\
0 & \varepsilon_{2}{ }^{-1} \mathbf{I}
\end{array}\right], \quad \mathbf{I}=\left[\begin{array}{ll}
1 & 0 \\
0 & 1
\end{array}\right], \\
& \mathbf{V}_{t}=\left[\begin{array}{lll}
\operatorname{Re} \mathbf{v}_{t 1}, & -\operatorname{Im} \mathbf{v}_{t 1}, \quad \operatorname{Re} \mathbf{v}_{t 2}, \quad-\operatorname{Im} \mathbf{v}_{t 2}
\end{array}\right] .
\end{aligned}
$$

If the injected beam is not matched to the ring lattice the decoherence of beam envelope oscillations leads to the emittance growth. After few thousand turns the system comes to equilibrium, and the emittance of the injected beam is determined by the following equation,

$$
\varepsilon_{i}^{\prime}=\frac{1}{8 \pi^{2} \varepsilon_{1} \varepsilon_{2}} \int d x^{4}\left(\mathbf{v}_{i}{ }^{+} \mathbf{U} \mathbf{x}\right)^{2} \exp \left(-\frac{1}{2} \mathbf{x}^{T} \boldsymbol{\Xi} \mathbf{x}\right), \quad i=1,2 .
$$

For initially uncoupled beam characterized by $\beta_{x}, \alpha_{x}, \beta_{y}$ and $\alpha_{y}$ that yields

$$
\begin{aligned}
& \varepsilon_{1}^{\prime}=\varepsilon_{1} A_{11}+\varepsilon_{2} A_{12}, \\
& \varepsilon_{2}^{\prime}=\varepsilon_{1} A_{21}+\varepsilon_{2} A_{22}, \\
& A_{12}=\frac{1}{2}\left(\frac{\beta_{y}}{\beta_{1 y}}\left[u^{2}+\alpha_{1 y}{ }^{2}\right]+\frac{\beta_{1 y}}{\beta_{y}}\left[1+\alpha_{y}{ }^{2}\right]-2 \alpha_{1 y} \alpha_{y}\right), \\
& A_{11}=\frac{1}{2}\left(\frac{\beta_{x}}{\beta_{1 x}}\left[(1-u)^{2}+\alpha_{1 x}{ }^{2}\right]+\frac{\beta_{1 x}}{\beta_{x}}\left[1+\alpha_{x}{ }^{2}\right]-2 \alpha_{1 x} \alpha_{x}\right), \\
& A_{21}=\frac{1}{2}\left(\frac{\beta_{x}}{\beta_{2 x}}\left[u^{2}+\alpha_{2 x}{ }^{2}\right]+\frac{\beta_{2 x}}{\beta_{x}}\left[1+\alpha_{x}^{2}\right]-2 \alpha_{2 x} \alpha_{x}\right), \\
& A_{22}=\frac{1}{2}\left(\frac{\beta_{y}}{\beta_{2 y}}\left[(1-u)^{2}+\alpha_{2 y}{ }^{2}\right]+\frac{\beta_{2 y}}{\beta_{y}}\left[1+\alpha_{y}{ }^{2}\right]-2 \alpha_{2 y} \alpha_{y}\right) .
\end{aligned}
$$

The developed Tevatron optics model is based on the results of differential optics measurements and takes coupling into account. In addition to coherent skew-quadrupole component in dipoles it also includes a few dozens local focusing and coupling errors scattered through the entire ring. Table 1 presents coupled Twiss parameters predicted by the model (see Section 2). Substituting these parameters into Eq. (8) yields that the emittance growth due to coupling was about $15 \%$ for each of two transfers before shimming of dipoles. This value dropped to about 3\% after the shimming of dipoles.

\section{Optics measurements and correction}

Optics correction have been complicated by limited time for optics measurements and the poor performance of the 30-year-old beam position monitors (BPM), in particular, by their low accuracy ( $\sim 150 \mu \mathrm{m}$ rms resolution) and malfunctioning ring-wide turn-by-turn mode. Presently, the only reliable way for optics measurements is the differential optics measurements, i.e. the orbit response to a single corrector bump. During last two years we mainly used the fast measurements, where only four correctors and an energy change are exercised. In this case the measurement takes about 5 minutes and getting time for the measurements is not a problem. The 
measurements are fully automated. Software records the reference orbit and its change in both planes due to a perturbation. Two correctors in each plane are chosen so that the betatron phase advance between them would be close to $\pi / 2$. Such set of measurements provides minimum information sufficient to restore all details of linear details. Off-line analysis yielded quad and skew-quad optics corrections matching the measured and computed orbits. The accuracy of the model has been tested by measurements of tune shifts due to small focusing change of a few single power supply quads. An agreement within $\sim 15 \%$ has been found for beta-function measurements. Comparatively large discrepancy is related to the fact that Tevatron tunes $(Q x=20.585, Q y=20.575)$ are quite close to half integer resonance. That additionally amplifies effects of optics errors on the beta-functions.

Data analysis at injection yielded the following results. There is a systematic difference between main bus superconducting dipoles and quads. As compared to 30 year old magnetic measurements the quads are $\sim 0.15 \%$ stronger. There is afore mentioned systematic skew-quad field in dipoles, $\mathrm{G}_{\mathrm{s}} \mathrm{A} / \mathrm{B}_{0} \sim 1.4 \cdot 10^{-4}$ for $\mathrm{A}=2.54 \mathrm{~cm}$. It is in good agreement with the measured displacement of coils, $\sim 150 \mu \mathrm{m}$. There are significant non-systematic (point-like) focusing and skew-focusing errors scattered through the entire machine. We used 30 quad and/or skew-quad corrections with strengths [0.5 - 2\%] of the main bus quad strength. The most striking finding was that focusing errors are about $0.5 \%$ for the final focus quadrupoles.

Similar to the injection we performed optics measurements for collision optics (low beta), where errors of focusing in the interaction region quads dominate errors in the sectors. The following conclusions were drawn out. There is a systematic difference between main bus superconducting dipoles and quads $(\sim 0.18 \%)$ consistent with measurements at the injection energy. Systematic skew-quad field in dipoles are $G_{s} A / B_{0} \sim 2.1 \cdot 10^{-4}$ for $A=2.54 \mathrm{~cm}$ which is 1.5 times larger than at injection. The origin of this discrepancy is unknown. Similar to the injection energy there are significant non-systematic (point-like) focusing and skew-focusing errors scattered throughout the entire machine. Although there is good correlation for large optics errors it is clearly different in details. Interaction region quads need to be fudged up to $1 \%$. It is well above what one would expect. Additionally, there is about $0.1 \%$ difference for quads of the same design. Model exhibited that due to optics mismatches the beta-functions in IPs were $~ 30 \%$ above design value and there was significant betatron function mismatch through entire machine.

Optics measurements proved that the resonance perturbation of beta-function dominated other optics discrepancies. Taking into account that analysis of differential orbit data is slow for on-line optics correction we used the optics measurements based on the measurements of tune shifts due to strength changes of one of 4 designated quads while the optics correction was performed using 4 other quads. Injection optics was corrected in 2003. The first step of the collision optics correction was carried out to correct optics mismatches in the IPs but there is still considerable mismatch in arcs.

\section{Extended differential orbit measurements}

Although the differential optics measurements are fast, data analysis is tedious and the results are not sufficiently accurate. Recently we introduced the extended measurements using LOCO (Linear Optics from Closed Orbit) technique ${ }^{[1,2,3]}$, where the single corrector orbit bumps are produced using about half of available correctors. That large redundancy in the data results in significant improvement of optics model accuracy. In this case a single measurement takes much longer ( 1 - 2 hour) and getting time for measurements is a problem but it is paid by improvement of the machine model. The effort has been built as collaboration between FNAL and ANL and 
aimed to upgrade the ANL software developed for $\mathrm{APS}^{[3]}$. The major objectives are software integration with FNAL data structures and optics software, taking into account strong X-Y coupling, and fitting data of dispersion measurements.

The idea of the method is to acquire large amount of data so that the systematic errors in the measurements could be determined. The unknowns are strengths and rolls of quads, strengths and rolls of correctors, and BPM responses and rolls. That amounts to about 800 unknowns. To have sufficient redundancy we perform measurements with $\sim 100$ correctors which yields $\sim 20,000$ equations. The singular value decomposition ${ }^{[4]}$ (SVD) is used to find unknowns from the measured data.

The first results of data analyses have been recently obtained. Further work is required to finish the project. Data analysis performed for collision optics yielded significant improvement of the fit accuracy. Compared to the model obtained before with differential orbit measurements the rms difference between measured and predicted differential orbits has been decreased from $10 \%$ to $1.5 \%$ of orbit oscillation amplitude. Figure 1 presents the computed errors for differential $\mathrm{BPM}$ response and corrector calibration errors.
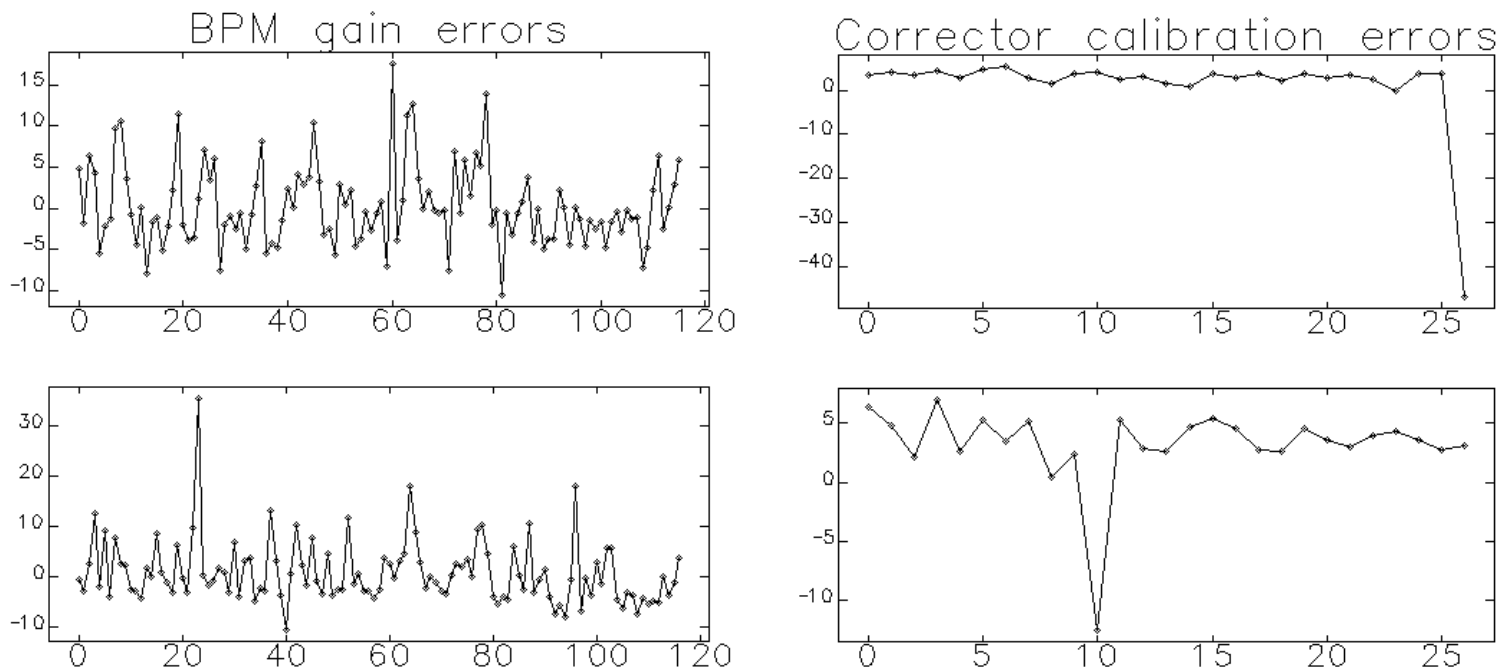

Figure 1. Computed errors (in percent) for differential BPM response (left) and corrector calibration errors (right); top pictures correspond to the horizontal plane, and bottom pictures to the vertical plane.

\section{Conclusions}

We built a reliable model of Tevatron optics based on the differential orbit measurements. The model includes actual power supply currents and has global and local focusing corrections to match the model to the measurements. It has been used to correct Tevatron optics and X-Y coupling. That resulted in a smaller emittance growth at injection ( $10-15 \%)$, and reducing betafunctions in IPs from $\sim 45 \mathrm{~cm}$ to the design values of $35 \mathrm{~cm}$. These optics corrections contributed to collider luminosity growth of $\sim 20-30 \%$. Presently, further improvements of the model are limited by poor accuracy of BPMs and sufficiently long measurement time. To address it we are upgrading BPM electronics. That will boost BPM accuracy from $\sim 150 \mu \mathrm{m}$ to $20 \mu \mathrm{m}$ rms and will allow to acquire turn-by-turn data for all BPMs. Turn-by-turn measurements will significantly reduce the measurement time, but their effective use implies development of software to fully 
utilize redundancy in their data. In addition the turn-by-turn measurements will allow us to measure lattice non-linearities.

Acknowledgements. The authors are grateful to Yu. Alexahin, V. Shiltsev and M. Syphers for multiple discussions and support. We also would like to thank the entire Tevatron department for help in carrying out of the measurements. One of the authors (VS) also would like to thank K.-J.Kim and K. Harkay for stimulating this work and support.

\section{References}

1. W. J. Corbett, M. Lee and V. Ziemann, PAC"93, Washington, DC, p. 108, 1993.

2. J. Safranec, Nucl. Inst. And Meth., A388, 27 (1997).

3. V. Sajaev, L. Emery, EPAC"02, Paris, France, p. 742, 2002.

4. W. H. Press, B. P. Flannery, S. A. Teukolsky, W. T. Vetterling, Numerical Recipes, Cambridge University Press, 1986. 